\title{
Selective adsorption and patterning of Si nanoparticles fabricated by laser ablation on functionalized self-assembled monolayer
}

\author{
K. Hata, ${ }^{\text {a) }}$ M. Fujita, S. Yoshida, S. Yasuda, T. Makimura, K. Murakami, \\ and $\mathrm{H}$. Shigekawa ${ }^{\mathrm{b}}$ ) \\ Institute of Applied Physics and CREST, Japan Science and Technology Corporation (JST), \\ University of Tsukuba, Tennodai 1-1-1, Tsukuba 305-8573, Japan \\ W. Mizutani and H. Tokumoto \\ Joint Research Center for Atom Technology, National Institute for Advanced Interdisciplinary Research, \\ Higashi 1-1-4, Tsukuba 305-8562, Japan
}

(Received 20 March 2001; accepted for publication 4 June 2001)

\begin{abstract}
We demonstrate an in situ selective adsorption of Si nanoparticles fabricated by laser ablation on a functionalized self-assembled monolayer (SAM). Si nanoparticles adsorbed on $-\mathrm{CH}_{3}$ terminated a SAM while Si particles did not adsorb on $-\mathrm{NH}_{2},-\mathrm{F},-\mathrm{OH}$, and $-\mathrm{COOH}$, terminated SAMs. The end group of a SAM solely determines the selectivity against Si nanoparticle adsorption. We utilized the screening ability of functionalized SAMs to pattern Si nanoparticles onto desired locations on a Si substrate. (C) 2001 American Institute of Physics. [DOI: 10.1063/1.1388025]
\end{abstract}

Within the emerging paradigm of nanotechnology, artificial nanostructures such as nanoparticles, nanotubes, and self-assembled monolayers (SAMs) are considered to offer great potential as basic building blocks to realize nanodevices and molecular electronics. In order to fulfill this goal, it would be necessary to develop technologies to assemble artificial nanostructures into highly functional complexities of the next level. One promising way to immobilize, assemble, and pattern nanostructures is to control the interaction between the nanostructures and substrate by changing their chemical functionality. ${ }^{1-6}$ SAM is an ideal candidate for this purpose because it possess several promising properties including: (1) a wide range of addressable terminating end groups that provides surfaces with various chemical identities, (2) a variety of substrates possible to fabricate a SAM and (3) the ability to pattern a chemically functionalized SAM by the microcontact printing $(\mu \mathrm{CP})$ method. ${ }^{7,8}$ The key point to successfully assemble and pattern nanostructures on a functionalized SAM is to achieve selective adsorption, i.e., to find a complementary pair of SAMs with different chemical end groups, where the target nanostructure does adsorb on one SAM while it does not on the other. While most of the previous research has pursued combinations of materials that stick together, searching combinations of materials that do not, is also a critical factor in achieving selective adsorption. One general route to achieve selective adsorption is to utilize the electrostatic interaction between oppositely charged nanomaterials and SAMs. ${ }^{4-6}$ In this case, selective adsorption can be readily achieved by changing the polarity of a SAM. Another way is to fabricate textured surfaces with different wettabilities, where nanomaterials preferably adsorb on regions with a specific wettability. ${ }^{3}$ In these methods,

${ }^{a)}$ Present address: Department of Chemistry and Chemical Biology, Harvard University, Cambridge, MA 02138; electronic mail:

khata@cmliris.harvard.edu

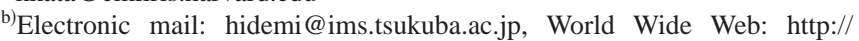
dora.ims.tsukuba.ac.jp. nanomaterials are suspended in a fluid and transferred to the surface, thus, intrinsically, the methods are wet processes. Utilizing these approaches, selective adsorption and subsequently patterning have been achieved on molecules, ${ }^{3}$ semiconductor nanowires, ${ }^{4}$ and metal particles ${ }^{3,5,6}$ so far.

Here we report on the in situ selective adsorption of $\mathrm{Si}$ nanoparticles fabricated by laser ablation ${ }^{9-11}$ on a functionalized SAM. The Si nanoparticle is a very interesting and important nanomaterial ${ }^{12-14}$ possesses many promising properties, e.g., it shows visible photoluminescence more efficient than in normal $\mathrm{Si}$ due to the quantum confinement effect. Different from earlier reports, selective adsorption of $\mathrm{Si}$ nanoparticles on a SAM we report here is achieved in a dry ambient and interestingly the driving force for adsorption is neither electrostatic interaction nor caused by different wettabilities of the SAM surface.

SAM substrates were loaded into a laser ablation chamber filled with Ar gas. A pulsed laser was focused and irradiated to a target $\mathrm{Si}$ wafer that was placed on the same plane of a SAM substrate [inset of Fig. 1(b)]. By laser ablation, it is possible to fabricate pure, well characterized, and relatively uniform, Si nanoparticles. ${ }^{9-11}$ Si nanoparticles formed by the collision of the hot $\mathrm{Si}$ atoms in the plasma with the inert gas are supposed to land on the SAM surface. Figure 1 shows typical scanning tunneling microscopy (STM) images of two SAM surfaces with different end groups taken after laser ablation. Laser ablation was carried out concurrently in a configuration shown in the inset of Fig. 1(b). White protrusions that did not exist before ablation were observed on $-\mathrm{CH}_{3}$ terminated SAM [Fig. 1(a)]. We confirmed that the white protrusions are $\mathrm{Si}$ nanoparticles through an extensive series of STM, atomic force microscopy (AFM), and photoluminescence experiments. ${ }^{15}$ The average size of Si nanoparticles was $7 \mathrm{~nm}$ when the Ar ambient is 5 Torr, while the size could be readily controlled, in the region where quantum confinement effect is important, by changing the Ar ambient pressure. Photoluminescence measurements showed an emission peak centered at $623 \mathrm{~nm}$, a value that is in the range of 

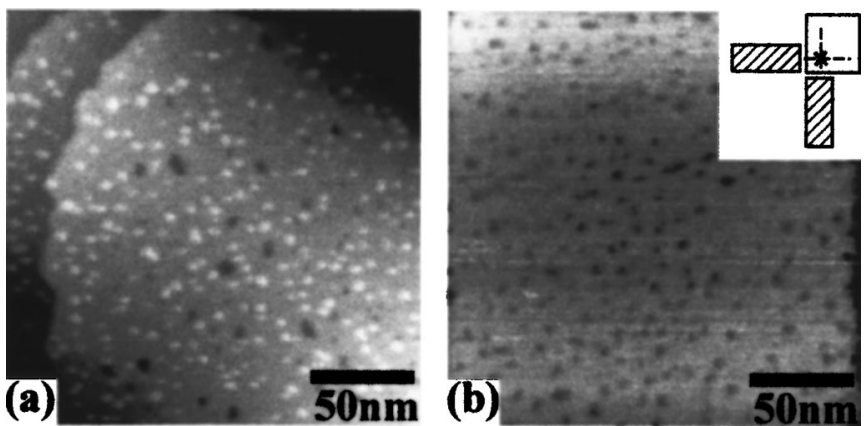

FIG. 1. Selective adsorption of Si nanoparticles on a functionalized SAM. (a) A STM image of a $-\mathrm{CH}_{3}$ (hexanethiol) terminated SAM and (b) a STM image of an - $\mathrm{COOH}\left[\mathrm{HS}\left(\mathrm{CH}_{2}\right)_{10} \mathrm{COOH}\right]$ terminated SAM, after laser ablation, recorded with tunneling current $=0.5 \mathrm{nA}$ and surface voltage $=500$ $\mathrm{mV}$. No eminent dependence on tunneling conditions was observed. Ablation conditions: Ar ambient pressure 5 Torr, shot number of pulsed laser 100. SAMs were prepared on $\mathrm{Au}(111) / \mathrm{mica}$ (thickness $50 \mathrm{~nm}$ ) by adsorption of thiols from millimolar solutions in ethanol for $12-24 \mathrm{~h}$, then rinsed with ethanol and dried under a flow of $\mathrm{N}_{2}$. Inset shows configuration of the SAM substrates (hatched rectangles) and the target $\mathrm{Si}$ wafer (square) during laser ablation. This configuration ensures that the laser ablation is carried out on the same condition for the two SAM substrates. The cross indicates the ablation spot. Typical distance from the ablation spot to the SAM substrates was approximately $5 \mathrm{~mm}$. The laser ablation chamber was filled with Ar gas of 2-10 Torr. Yttrium-aluminum-garnet pulsed laser shots (10 ns, $25 \mathrm{~mJ})$ were focused and irradiated to the Si target at frequencies of 1 or $10 \mathrm{~Hz}$.

the characteristic emission wavelengths of $\mathrm{Si}$ nanoparticles fabricated by our laser ablation conditions. Si nanoparticles were firmly attached to a SAM: they were not removed by ethanol rinsing, and remained stable for at least a couple of months. These features would be preferred in any practical use, and would enable one to implement many sequential processes to fabricate more complex nanoarchitectures. In contrast, no Si nanoparticles were observed on a $-\mathrm{COOH}$ terminated SAM [Fig. 1(b)]. The screening effect of a $-\mathrm{COOH}$ terminated SAM (and others described later) to the adsorption of Si nanoparticles was complete, and not a single Si nanoparticle was observed on the surface regardless of the position and for many experimental runs carried out on various laser ablation conditions.

To elucidate the adsorption mechanism of Si nanoparticles on a SAM, we carried out laser ablation on several SAMs that have different end groups with various polarities and wettabilities. Each time, as a reference, laser ablation was concurrently carried out on a $-\mathrm{CH}_{3}$ terminated SAM substrate in a configuration shown in Fig. 1(b). Results are listed in Table I. Interestingly, we found that $\mathrm{Si}$ nanoparticles only adsorb on a $-\mathrm{CH}_{3}$ terminated SAM while $\mathrm{Si}$ particles do not absorb on $-\mathrm{NH}_{2},-\mathrm{F},-\mathrm{OH}$, and $-\mathrm{COOH}$ terminated SAMs. Since Si nanoparticles did not adsorb on SAMs with

TABLE I. Adsorption characteristics of various functionalized SAMs against Si nanoparticles listed with the polarity and wettability properties of the end group of SAMs. Functionalized SAMs were fabricated from $\mathrm{HS}\left(\mathrm{CH}_{2}\right)_{8} \mathrm{OH}, \mathrm{HS}\left(\mathrm{CH}_{2}\right)_{10} \mathrm{COOH}, \mathrm{HS}\left(\mathrm{CH}_{2}\right)_{8} \mathrm{NH}_{2}$, and $\mathrm{HS}\left(\mathrm{CF}_{2}\right)_{7}\left(\mathrm{NH}_{2}\right)_{2} \mathrm{CF}_{3}$.

\begin{tabular}{lccc}
\hline \hline End group & Si particle ? & Polarity & Hydro- \\
\hline$-\mathrm{CF}_{3}$ & $\times$ & - & Phobic \\
$-\mathrm{CH}_{3}$ & $\bullet$ & 0 & Phobic \\
$-\mathrm{NH}_{2}$ & $\times$ & + & Philic \\
$-\mathrm{OH}$ & $\times$ & - & Philic \\
$-\mathrm{COOH}$ & $\times$ & - & Philic \\
\hline
\end{tabular}
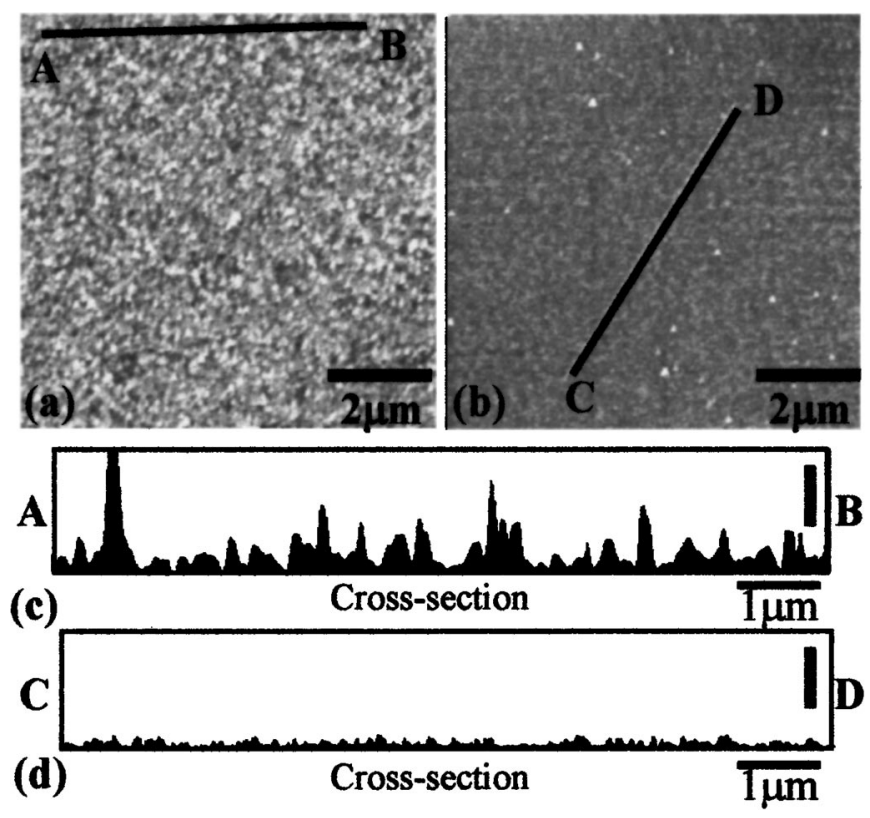

FIG. 2. Selective adsorption of Si nanoparticles on a functionalized $\mathrm{SAM} / \mathrm{Si}$ and Si. (a) An AFM image of an oxidized Si surface and (b) an AFM image of an $-\mathrm{NH}_{2}$ terminated SAM fabricated on a Si substrate, both taken after laser ablation. (c) Cross section of $\mathrm{AB}$ showing adsorption of Si nanoparticles. (d) Cross section of $\mathrm{CD}$, no Si nanoparticles adsorbed. The scale bars in (c) and (d) correspond to $4 \mathrm{~nm}$.

both negative and positive charged end groups, while did adsorb on a $-\mathrm{CH}_{3}$ terminated SAM that has a neutral polarity, we exclude electrostatic interaction as the driving force of adsorption. Another possibility is that hydrophobic Si nanoparticles might feel an attractive force to the hydrophobic $-\mathrm{CH}_{3}$ terminated SAM provided an ultrathin water film exists on the surface. However, we rule out this possibility since (1) no Si nanoparticles adsorbed on the most hydrophobic $-\mathrm{F}$ terminated SAM, and (2) no increase of Si nanoparticle adsorption was observed on a $-\mathrm{CH}_{3}$ terminated SAM intentionally exposed to water ambient before laser ablation. Our results indicate that neither electrostatic interaction nor different wettabilities of the SAM surfaces are responsible for the selective adsorption characteristics of Si nanoparticles on a functionalized SAM. More investigations are required to sort out the mechanism.

We confirmed that the length of the alkyl chain has nothing to do with the selective adsorption of Si nanoparticles. The Si nanoparticles did adsorb on $-\mathrm{CH}_{3}$ terminated SAMs that have different lengths of alkyl chains (pentanethiol, hexanethiol, and octanethiol), while Si particles did not adsorb on SAMs terminated with other end groups regardless the lengths of alkyl chains (Table I). This result strongly implies that the end group solely determines the selective adsorption characteristic. To explore this aspect further, we turned to the technologically more important $\mathrm{Si}$ substrate, and sought whether selective adsorption could be achieved there. As Si nanoparticles easily and firmly adhere to the native oxide layer on $\mathrm{Si}$, here we focused on the screening property of functionalized SAMs. We carried out laser ablation on an $-\mathrm{NH}_{2}$ terminated SAM substrate [Fig. 2(b)] and on another oxidized Si substrate [Fig. 2(a)], concurrently. Both surfaces were atomically flat before ablation. After ablation, photoluminescence measurements showed a spectrum similar to that Downloaded 24 Dec 2009 to 130.158.56.186. Redistribution subject to AIP license or copyright; see http://apl.aip.org/apl/copyright.jsp 


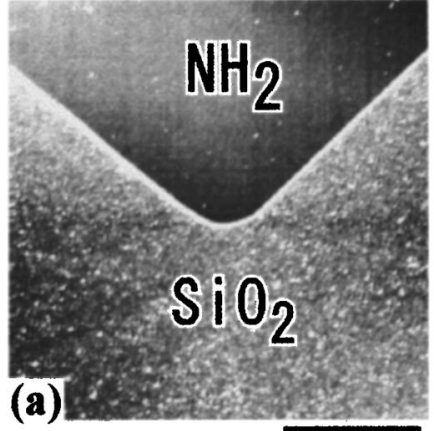

$\mathbf{5 \mu m}$

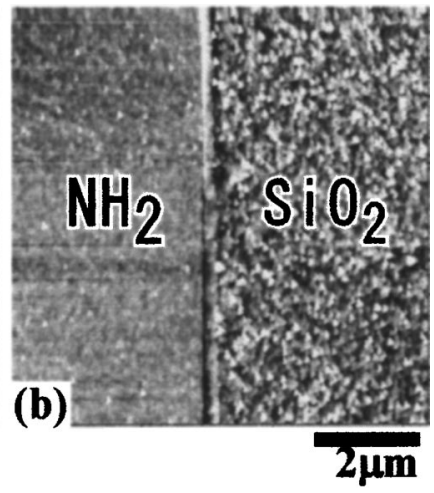

FIG. 3. Patterning of Si nanoparticles. (a) and (b) AFM images demonstrating a successful patterning of Si nanoparticles. Patterned SAMs were fabricated using patterned polydimethylsiloxane (PDMS) stamps that were made by the method described in Ref. 8. Si wafers were cleaned in a $\mathrm{H}_{2} \mathrm{SO}_{4}: \mathrm{HNO}_{4}=4: 1$ solution for $30 \mathrm{~min}$. A PDMS stamp inked with 3-(aminoethylamino)propyltrimethoxysilane (solvent hexane) was brought into contact with cleaned $\mathrm{Si}$ substrates for 30 min with a mass of $\sim 0.5 \mathrm{~kg}$ on top of the stamp-substrate system under $\mathrm{N}_{2}$ atmosphere. The patterned SAM-Si substrate was characterized by AFM prior to laser ablation.

observed for $\mathrm{Si}$ nanoparticles on $-\mathrm{CH}_{3}$ terminated SAMs. AFM images and their cross sections [Figs. 2(c) and 2(d)] show protrusions on the oxidized $\mathrm{Si}$ substrate that did not exist before laser ablation, and we attribute them to Si nanoparticles. On the other hand, almost no Si nanoparticles were observed on an $-\mathrm{NH}_{2}$ terminated SAM, and the surface remained atomically flat as before ablation. These results strongly indicate that the selective adsorption characteristic is determined solely by the terminating end group of a SAM, a point that would be of extreme importance for any future practical use and that highlights the generality of our approach.

We utilized this screening ability of functionalized SAMs to pattern Si nanoparticles onto desired locations. The key point here is to use the $\mu \mathrm{CP}$ method to fabricate a pattered SAM on an oxidized Si substrate, whereby Si nanoparticles selectively adhere to the bare oxidized Si regions while they are screened at regions covered with a functionalized
SAM. Figure 3 shows AFM images (after laser ablation) of a Si substrate on which a patterned $-\mathrm{NH}_{2}$ terminated SAM was fabricated by the $\mu \mathrm{CP}$ method. ${ }^{6,7} \mathrm{Si}$ nanoparticles solely adsorbed on the oxidized Si regions and not on regions covered with a SAM, a result that demonstrates a successful patterning of Si nanoparticles.

In conclusion, we found that $\mathrm{Si}$ nanoparticles selectively adsorb on functionalized SAMs. The selective adsorption characteristic is determined solely by the end group of a SAM, thus a similar selective adsorption should be obtainable on many different substrates. Significantly, this selective adsorption is achieved in the dry ambient during laser ablation, and we believe that the wide range of available SAMs combined with the wide variety of nanostructures possible to fabricate by laser ablation would open up an opportunity to assemble these nanomaterials into high functional complexities of the next level.

The support of a Grant-in-Aid for Scientific Research from the Ministry of Education, Science and Culture of Japan is acknowledged.

${ }^{1}$ S. R. Whaley, D. S. English, E. L. Hu, P. F. Barbare, and A. M. Belcher, Nature (London) 405, 665 (2000).

${ }^{2}$ A. K. Boal, F. Iihan, J. E. DeRouchey, T. T. Albrecht, T. P. Russel, and V. M. Rotello, Nature (London) 404, 746 (2000).

${ }^{3}$ M. Gleiche, L. F. Chi, and H. Fuchs, Nature (London) 403, 173 (2000).

${ }^{4}$ Y. Huang, X. Duan, Q. Wei, and C. M. Lieber, Science 291, 630 (2001).

${ }^{5}$ H. X. He, H. Zhang, Q. G. Li, T. Zhu, S. F. Y. Li, and Z. F. Liu, Langmuir 16, 3846 (2000).

${ }^{6}$ J. Tien, A. Terfort, and G. M. Whitesides, Langmuir 13, 5349 (1997).

${ }^{7}$ A. Kumar and G. Whitesides, Appl. Phys. Lett. 63, 2002 (1993).

${ }^{8}$ P. M. St. John and H. G. Craighead, Appl. Phys. Lett. 68, 1022 (1996).

${ }^{9}$ E. Werwa, A. A. Seraphin, L. A. Chiu, C. Zhou, and K. D. Kolenbrander, Appl. Phys. Lett. 64, 1821 (1994).

${ }^{10}$ T. Makimura, Y. Kuni, and K. Murakami, Jpn. J. Appl. Phys., Part 2 35, L735 (1996).

${ }^{11}$ T. Makimura, T. Sakamoto, and K. Murakami, Appl. Phys. Lett. 76, 1401 (2000).

${ }^{12}$ L. T. Canham, Appl. Phys. Lett. 57, 1046 (1990).

${ }^{13}$ B. Gelloz and N. Koshida, J. Appl. Phys. 88, 4319 (2000).

${ }^{14}$ M. O. Watanabe, T. Miyazaki, and T. Kanayama, Phys. Rev. Lett. 81, 5362 (1998).

${ }^{15} \mathrm{~K}$. Hata (unpublished). 